\title{
Birefringence of Rubber during Creep and Recovery
}

\author{
P. H. Mott and C. M. Roland*
}

Chemistry Division, Code 6120, Naval Research Laboratory, Washington, D.C. 20375-5342

Received J une 11, $1996^{\otimes}$

\begin{abstract}
Measurements of creep and recovery of lightly cross-linked polybutadiene, carried out in both shear and compression geometries, reveal two deviations from the stress optical law: the birefringence increases during the constant stress creep, and the birefringence is not zero during the stress-free recovery. Most interestingly, the sign of the birefringence during recovery was found to be opposite to that during creep. This implies that the chain segments have a net orientation opposite to the macroscopic deformation.
\end{abstract}

\section{Introduction}

Investigations of the mechanical behavior of polymers sometimes take advantage of the stress optical law, relating the true stress, $\sigma$, to the birefringence $\Delta \mathrm{n}^{1-3}$

$$
\Delta \mathrm{n}=\mathrm{C} \sigma
$$

The stress optical coefficient, $C$, is generally considered to remain constant during deformation. According to constraint models of rubber elasticity, C has a weak dependence on stress; ${ }^{4-6}$ however, the quantitative accuracy of these models is limited. ${ }^{7}$ Experiments indicate the stress optical law to remain valid up through at least moderate extensions (e.g., 50\%) for a wide variety of neat rubbers in mechanical equilibrium. ${ }^{4,8-13}$ Equation 1 also is reputed to hold in the nonlinear viscoelastic regime. ${ }^{2}$ Dynamic measurements on polymers far above and well below the glass transition temperature have been interpreted in terms of a $\mathrm{C}$ that is independent of stress and test frequency. ${ }^{14-22}$

In rubbers, a direct proportionality between stress and birefringence implies that both have the same physical origin-orientation of the segments comprising the polymer chains. ${ }^{2,3,23}$ Four departures from the stress optical law are known: (i) When the orientation of the bond vectors (responsible for the intrinsic birefringence) is no longer proportional to the orientation of the chain end-to-end vector (responsible for the mechanical stress). This occurs for the very large stresses, and consequent bond distortions, associated with finite chain extensibility. (ii) Behavior in the softening zone of the viscoelastic spectrum ostensibly violates the stress optical law; however, the stress optical coefficient obtained from dynamic mechanical experiments in this regime can be decomposed into a glassy and rubbery component, both conforming to eq 1. ${ }^{14-22}$ (iii) While the intrinsic birefringence is proportional to the stress, the form birefringence, arising from spatial variations of the dielectric constant, is not. Significant for phase-separated blends and semicrystalline polymers, 2,24 the form birefringence is negligible in homogeneous materials. (iv) "Doublenetwork" rubbers, formed by cross-linking under strain a previously prepared isotropic network, exhibit measurable birefringence at zero stress. ${ }^{25}$ Their behavior can be interpreted on the basis of the idea of two independent networks, one extended and the other in compression. ${ }^{26-29}$

\footnotetext{
${ }^{\otimes}$ Abstract published in Advance ACS Abstracts, November 15 , 1996.
}



Figure 1. Schematic of the apparatus used to simultaneously measure birefringence, stress, and strain during creep and recovery.

Changes in $C$ during creep and recovery were seen in poly(vinyl chloride), a glassy polymer..$^{30}$ A suggestion of departure from the stress optical law in rubber was reported by Saunders, ${ }^{9}$ who measured non-zero birefringence in un-cross-linked natural rubber during recovery from creep. These phenomena, which have not been investigated further, are the subject of the present study. Herein we describe bir efringence measurements during creep and recovery of cross-linked polybutadiene. Due to its random cis and trans copolymer structure, the material is incapable of crystallizing; this avoids a complication in the natural rubber experiments of Saunders. ${ }^{9}$ Deformations were carried out 100 deg above the glass transition temperature, so that any interference from a "glassy" stress optical coefficient ${ }^{14-22,30}$ is also avoided.

\section{Experimental Section}

The polymer was a high molecular weight polybutadiene (type 1209 from the Goodyear Tire \& Rubber Co.) having a predominantly 1,4-microstructure (35\% cis). The cross-linking agent, dicumyl peroxide, was incorporated using two-roll mill, followed by compression molding for $120 \mathrm{~min}$ at $150^{\circ} \mathrm{C}$. The cross-link density, cal culated from Young's modulus, ${ }^{3}$ was 220 $\mathrm{mol} / \mathrm{m}^{3}$; this means the molecular weight between cross-links was more than a factor of 2 larger than the molecular weight between entanglements for polybutadiene. ${ }^{31}$

A schematic of the experimental apparatus, which utilized a modified Dynastat Mark II instrument (Imass Corp.), is shown in Figure 1. The sample was mounted in a nitrogenfilled chamber, maintained for all experiments at $31{ }^{\circ} \mathrm{C}$. A constant load was applied and the displacement measured by using a linear voltage differential transducer (LVDT). Since 
the intensity of the HeNe laser fluctuated as much as $\pm 5 \%$ over periods of roughly $10 \mathrm{~min}$, it was necessary to normalize the measured signal by the incident beam intensity. The experimental data-load, displacement, incident and transmitted light intensity-was acquired as a function of log time, beginning at 0.1 s intervals. After 80 s the time intervals were long enough to allow signal averaging over $1 / 60 \mathrm{~s}$, reducing the experimental noise.

Both shear experiments on rectangular specimens $(12.2 \mathrm{~mm}$ $\times 12.2 \mathrm{~mm} \times 17.8 \mathrm{~mm}$ ) and compression of cylindrical samples (12.2 $\mathrm{mm}$ in diameter and $17.8 \mathrm{~mm}$ high) were utilized. A simple shear geometry offers the advantage of invariant sample thickness, and hence path length for the laser beam, during creep. However, it was necessary to glue (cyanoacrylate adhesive) the films to the shear fixture, which causes residual stresses near the bonded area. Tall $(17.8 \mathrm{~mm})$ specimens were used to minimize this end effect and achieve a homogeneous state of stress in the area of measurement.

Most of the work employed a compression geometry, with the cylinders placed on a Teflon-coated stage, lightly coated with silicone oil. This ensured complete slippage of the faces during loading, as evidenced by an absence of bulging of the cylinder. Maintaining the cylindrical geometry avoids shear stresses along the contact surface. ${ }^{32-34}$ The advantage of the compression geometry is that the upper cross head fixture could be taken out of contact with the sample during recovery, thus ensuring that any birefringence during the recovery phase was not due to an experimental artifact such as load cell drift. However, the diameter of a nonbonded cylinder changes during deformation, so that the true stress is not constant. In the present experiments $\sigma$ decreases by as much as $6 \%$ during compressive creep. The creep compliance is the ratio of the strain to the true stress, $J=\epsilon / \sigma$, while the recoverable compliance, $J_{R}$, is the strain during recovery divided by the creep stress.

Typically, samples were allowed to creep for $60000 \mathrm{~s}$ (16.7 $\mathrm{h})$, followed by recovery for ca. $15000 \mathrm{~s}$. The birefringence $\Delta \mathrm{n}$ of the sample was calculated as

$$
\Delta \mathrm{n}=\frac{\lambda}{2 \pi \mathrm{l}} \arccos \left(1-\frac{2 \mathrm{I}}{\mathrm{I}_{\mathrm{m}}}\right)
$$

where $\lambda(=632.8 \mathrm{~nm})$ is the wavelength of the laser, I is the path length of the beam through the sample, $I$ is the transmitted light intensity (normalized by the incident intensity), and $I_{m}$ is the maximum normalized transmitted intensity. For compression, the path length is a function of the strain, I = $D_{0} /(1+\epsilon)^{1 / 2}$, where $D_{0}$ is the initial diameter. The sign of the birefringence was determined in separate experiments using a quarter-wave plate installed between the sample and the analyzer. In this method, the birefringence is measured by rotation of the analyzer polarizing filter to minimize the intensity of the transmitted light, ${ }^{1,32}$

$$
\Delta \mathrm{n}=\frac{\theta \lambda}{\pi l}
$$

where $\theta$ is the rotation angle of the analyzer.

\section{Results}

In Figure 2 we plot the birefringence measured for the polybutadiene for stresses in the range $-435 \mathrm{kPa}$ $>\sigma>+435 \mathrm{kPa}$, measured $8 \mathrm{~h}$ after application of the stress. Any additional creep beyond this time is immeasurably small, although equilibrium has probably not been attained. (Complete cessation of creep in lightly cross-linked rubbers requires extremely long time periods. ${ }^{35}$ ) The stress optical coefficient determined for the polybutadiene, $\mathrm{C}=3.58 \mathrm{GPa}^{-1}$, is constant within the experimental error; thus, for both tension and compression, the material conforms to the stress optical law when in a condition of mechanical equilibrium.

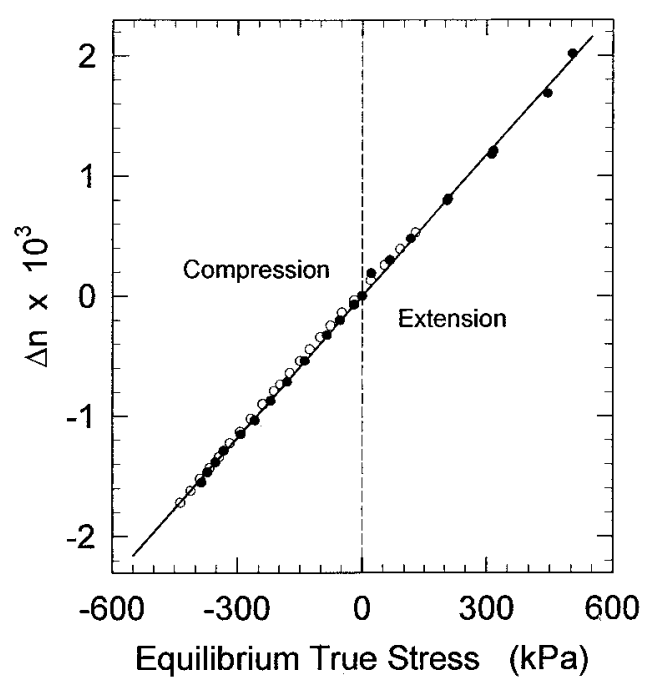

Figure 2. Optical birefringence as a function of true stress for the cross-linked polybutadiene. Hollow and solid symbols represent different samples. Linear regression yields the stress optical coefficient, $\mathrm{C}=3.6 \mathrm{GPa}^{-1}$.

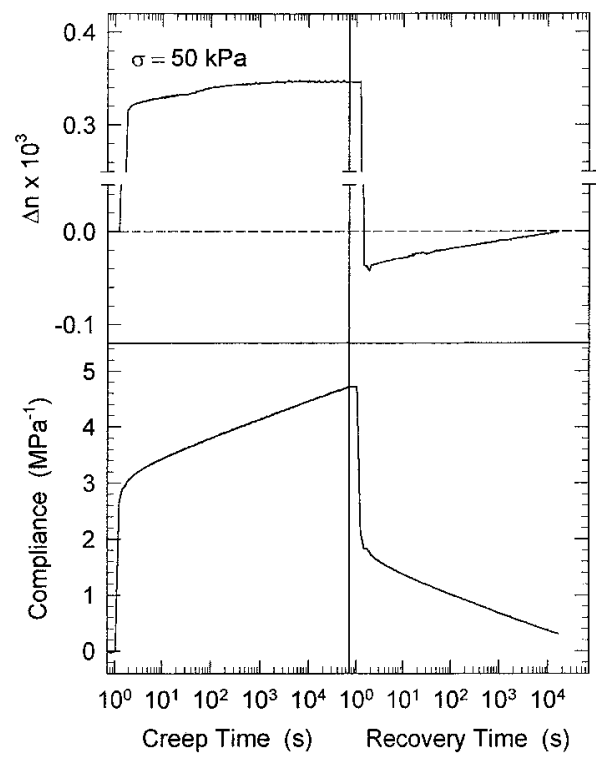

Figure 3. Creep compliance and creep recovery of the polybutadiene deformed in shear, along with the associated birefringence. The compliance never reaches steady state, although the birefringence becomes invariant after about $10^{4}$ $\mathrm{s}$. Note that the sign of $\Delta \mathrm{n}$ is negative during recovery.

Figure 3 shows typical results from a shear experiment, in which a $50 \mathrm{kPa}$ creep stress was applied for $60000 \mathrm{~s}$, followed by $10000 \mathrm{~s}$ of zero stress recovery. N ote that the birefringence during creep is not constant, despite the constancy of the stress. Furthermore, during the recovery phase, the birefringence is not zero, notwithstanding the absence of stress. Both phenomena are at odds with the stress optical law. More remarkable is the sign of the birefringence-during recovery it is opposite to that during creep. This implies orientation during recovery which is opposite to that during creep, even though the macroscopic deformation is the same for the two phases of the experiment. A similar effect was observed in glassy polymers subjected to cyclic loading. ${ }^{30}$

During the course of the creep, the compliance changes by about $53 \%$, which is an order of magnitude larger than the change in the birefringence. Similarly, the relative change in the compliance during recovery 


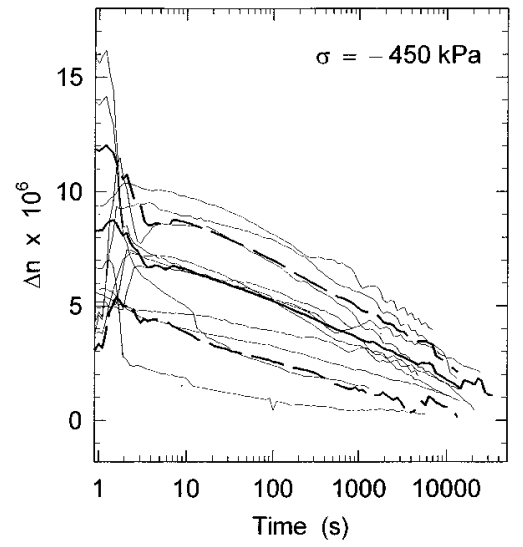

Figure 4. Birefringence during creep recovery of eleven, nominally identical, samples of the polybutadiene. The data were taken after $60000 \mathrm{~s}$ creep at the indicated compressive stress. The heavy solid line is the average and the two dashed lines represent the standard deviation.

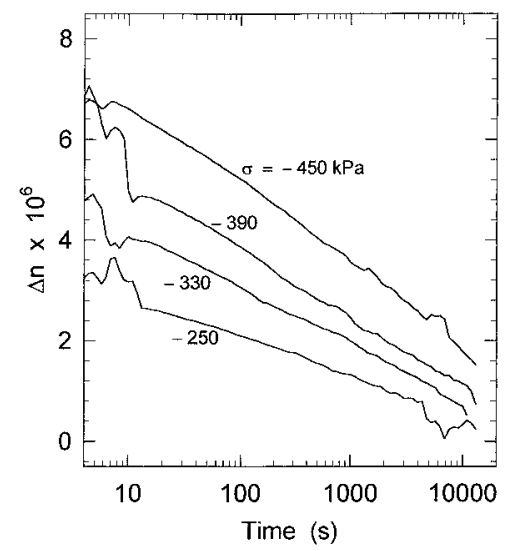

Figure 5. Birefringence in stress-free cylinders during re covery after $60000 \mathrm{~s}$ of creep at the indicated stresses. The data represent the average of 5-15 measurements each.

greatly exceeds that of the birefringence. After about $10000 \mathrm{~s}$, any changes in birefringence during either phase of the experiment become immeasurably small, even though the compliance has not yet reached steady state.

Although measurable birefringence was al ways present during recovery, its magnitude varied from experiment to experiment. To give some indication of this scatter, the bir efringence measured for eleven different samples, each recovering from $60000 \mathrm{~s}$ of creep at $\sigma=-450 \mathrm{kPa}$, is shown in Figure 4. The first few seconds of the recovery period reflect experimental artifacts, related to fast removal of the load. Beyond this, however, substantial scatter remains in the (weak) birefringence data; the standard deviation during recovery is about $30 \%$ of the mean birefringence. Such scatter is greater than can be accounted for from sample inhomogeneity or errors in the measurement technique. Because of this poor reproducibility, all results reported herein are the average of as many as 15 measured samples. Notwithstanding the variations in magnitude, the time scale of the birefringence decay was quite reproducible.

Figure 5 shows the birefringence during recovery for four levels of creep stress. The results indicate that the birefringence during recovery increases with the magnitude of the creep stress. The time required for $\Delta \mathrm{n}$ to reach steady state, however, appears to be constant.

During these experiments, creep strains as high as 0.25 were attained; thus, these experiments bring the

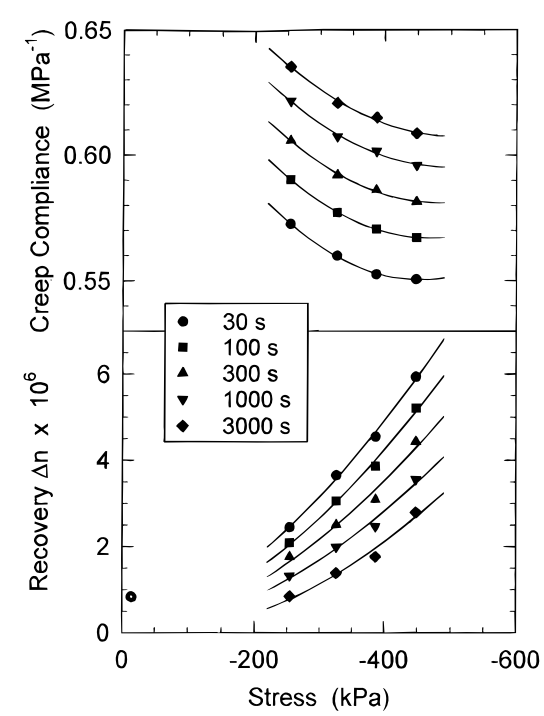

Figure 6. Isochronal creep compliance and isochronal birefringence during creep recovery (after $60000 \mathrm{~s}$ creep), as a function of the compressive stress.

material into the realm of nonlinear viscoelastic behavior. ${ }^{36}$ Large stresses facilitated measurement of the weak birefringence signal, but qualitatively equivalent results were obtained in experiments carried out at lower stresses. For example, at $14.5 \mathrm{kPa}$ compressive stress, creep strains of $1.4 \%$ were reached after 70000 $\mathrm{s}$, during which time the birefringence behaved in a fashion very similar to that seen in Figure 3. Birefringence was also detected during the recovery; for example, $\Delta \mathrm{n}=0.84 \times 10^{-6}$ at $30 \mathrm{~s}$, with a sign opposite to that during the creep. Thus, the deviations from the stress optical law observed herein are not a consequence of nonlinear viscoelastic behavior.

To illustrate the stress dependence of the recovery birefringence, we plot in Figure 6 the creep compliance and the residual birefringence, both taken at an arbitrary time during the recovery period, for different creep stresses. The data suggest that both quantities are nonlinear functions of the applied stress. The creep compliance is decreasing with increasing stress, which is typical elastic behavior. ${ }^{37-39}$ Also shown in Figure 6 is a recovery birefringence datum obtained after creep at low strain.

\section{Discussion}

The departures herein from the stress optical law were observed directly, without reliance on any calculations (such as to remove form birefringence or to apply Boltzmann superpositioning). Although moderately large deformations were employed, their only purpose was to amplify the phenomena. The changes in birefringence during creep and the non-zero birefringence during recovery were present in small strain experiments as well.

Since optical birefringence reflects anisotropy of the chain segments, any change in their orientation at constant (or zero) stress will alter the birefringence. Nevertheless, the literature is adamant concerning a direct connection between the stress and birefringence. ${ }^{1-3,24,40}$ This general (and evidently incorrect) expectation arises from the fact that models of chain elasticity yield respective expressions for the stress and birefringence having the same strain dependence. However, in a viscoelastic material, deformation of chains (or network strands) evolves over the course of the creep 


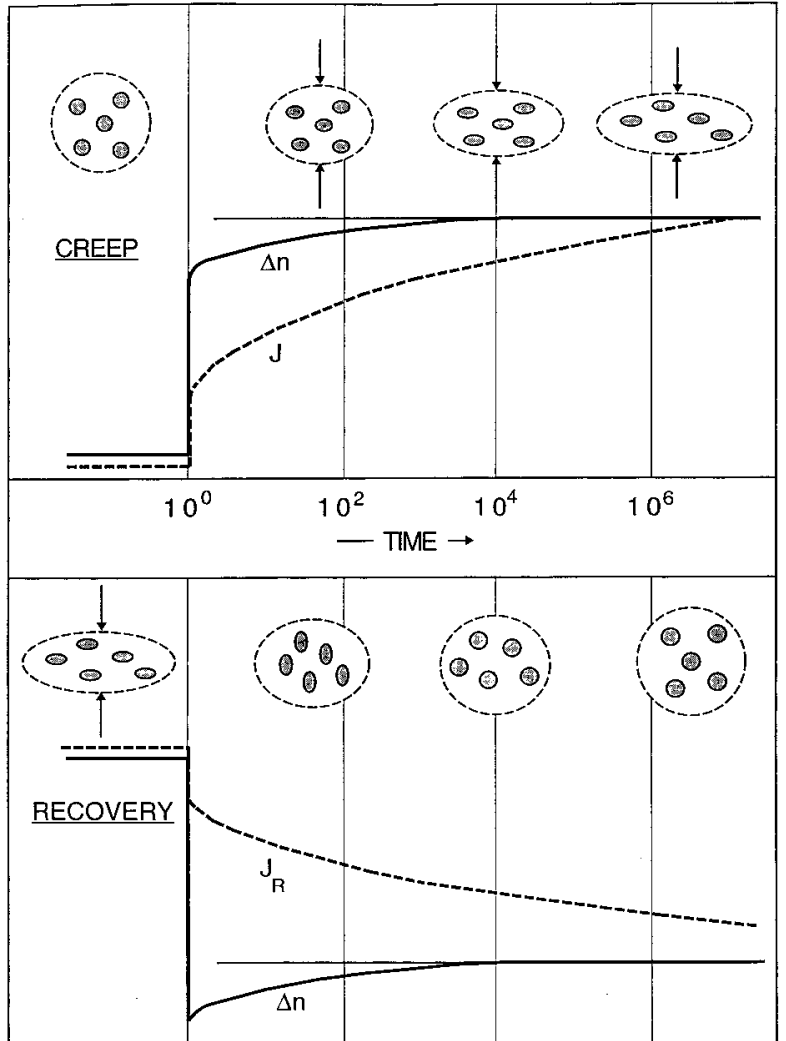

Figure 7. Schematic rationalizing the two processes responsible for respectively the birefringence and the strain during creep and recovery. The small circles correspond to the local segment orientation, governing the birefringence; the larger circles reflect the macroscopic strain. After $10^{4} \mathrm{~s}$, the birefringence has reached steady state, and so the small circles have attained an equilibrium deformation. The macroscopic deformation continues beyond $10^{4} \mathrm{~s}$ and involves rearrangement of the small elements.

period. This implies that the local segment orientation, and hence $\Delta \mathrm{n}$, will necessarily change during creep, notwithstanding the constancy of the stress.

More perplexing is the fact that $\Delta \mathrm{n}$ during recovery was found to be opposite in sign to that during creep. This implies two intriguing features concerning the segment orientation during recovery: (i) it is opposite to the segment orientation during creep and (ii) it corresponds to extension of the segments for a macroscopic strain which is compression. The latter point argues against any facile explanation of (i) based on a recoil effect. The origin of this anomalous recovery behavior is presently unknown, but at least a crude guide for further investigation can be suggested. As pointed out by Ferry, ${ }^{41}$ the point of zero stress in an extension-retraction cycle carried out at constant strain rate does not correspond to zero stored energy. He ascribed this to the fact that some chain elements are extended, while others are in compression, resulting in a balance of stresses, and hence macroscopic orientation in the absence of macroscopic stress. Similarly, the recovery process herein must involve competing orientations.

As seen in Figure 2, the birefringence during both creep and recovery assumes a constant value after roughly $10^{4} \mathrm{~s}$. This is more than a decade sooner than the time required for the compliance to reach steady state. The fact that the mechanisms underlying the birefringence and the strain occur on different time scales suggests the existence of orientation processes having different length scales. Depicted in Figure 7 is an idealized representation of these two processessegment orientation, giving rise to the birefringence, and a more delocalized process responsible for the macroscopic strain. What structural feature might be identified with this process, such as the entanglements or the network strands, is unknown. Figure 7 is a naive attempt to reconcile the fact that the segment orientation is opposite to the macroscopic deformation. This is an enigma, since these same segments comprise the structures having orientation over more distant length scales. It is not obvious, however, that distinct entities, identifiable by their orientation, can exist in an ostensibly homogeneous material. Previously, allusions have been made in the literature to structural heterogeneities of various sorts in neat polymeric materials, ${ }^{42-45}$ as well as to heterogeneous deformations, as seen in polymer gels. ${ }^{46,47}$

In conclusion, we mention an effect in rubber which may bear on the present phenomenon. Cross-linked rubber exhibits "linear" viscoelastic behavior-defined as direct proportionality between stress and strain, and time invariance of the mechanical response (referring to Boltzmann superpositioning, not viscoelasticity)-over a broader range of deformations than most materials. ${ }^{48}$ Nevertheless, there is a curious effect, known as Mullins softening in the rubber literature, ${ }^{36,49-53}$ which refers to the dissipation of mechanical energy in excess of that accounted for by linear relaxation. When subjected to reversing deformations, rubber is invariably found to exhibit larger mechancial hysteresis than predicted from application of the Boltzmann superposition principle. Hence, after a strain reversal, the stress is overestimated, even though "strict" linearity has been demonstrated over the same range of strains without a reversal. ${ }^{36,51-53}$ In polymeric liquids there is an analogous phenomenon, ${ }^{54-56}$ whereby the failure of constitutive equations (including nonlinear models) is seen for deformation histories involving a change in the sign of the strain.

The creep-recovery experiments, revealing herein the anomalous birefringence, are reversing strain deformations. However, we do not believe the phenomenon to be a consequence of nonlinear viscoelasticity. Qualitatively identical results were obtained in creep-recovery experiments involving small (ca. 1\%) deformations. Nevertheless, reversing strain deformations appear to be uniquely capable of revealing peculiar viscoelastic behavior, and as such present demanding, and presently unanswered, challenges for theory.

Acknowledgment. Stimulating conversations with D. J . Plazek are gratefully acknowledged. This work was supported by the Office of Naval Research. P.H.M. expresses his gratitude for a National Research Council/ Naval Research Laboratory postdoctoral fellowship.

\section{References and Notes}

(1) Riande, E.; Saiz, E. Dipole Moments and Birefringence of Polymers; Prentice Hall: Englewood Cliffs, NJ , 1992.

(2) Doi, M.; Edwards, S. F. The Theory of Polymer Dynamics; Clarendon Press: Oxford, U.K., 1986.

(3) Treloar, L. R. G. The Physics of Rubber Elasticity; Clarendon Press: Oxford, U.K., 1975.

(4) Erman, B.; Flory, P. J . Macromol ecules 1983, 16, 1601, 1607.

(5) Galiatsatos, V. Macromolecules 1990, 23, 3817.

(6) Kloczkowski, A.; Mark, J . E.; Erman, B. Comp. Polym. Sci. 1995, 5, 37.

(7) Mott, P. H.; Roland, C. M. Macromolecules 1996, 29, 6941.

(8) Treloar, L. R. G. Trans. Faraday Soc. 1947, 43, 284. 
(9) Saunders, D. W. Trans. Faraday Soc. 1956, 52, 1414.

(10) Smith, K. J .; Puett, D. J . Appl. Phys. 1966, 37, 346.

(11) Neaffer, R. O.; Galiatsatos, V. Macromolecules 1993, 26, 4013.

(12) Fukuda, M.; Wilkes, G. L.; Stein, R. S. J . Polym. Sci., Polym. Chem. Ed. 1971, 9, 1417.

(13) Ishikawa, T.; Nagai, K.J . Polym. Sci. Polym. Chem. Ed. 1969, 7, 1123; Polym. J. 1970, 1, 116.

(14) Read, B. E. Polym. Eng. Sci. 1983, 23, 835.

(15) Read, B. E. Polymer 1964, 5, 1.

(16) Read, B. E. J . Polym. Sci. C 1964, 5, 87.

(17) Read, B. E. J. Polym. Sci. C 1967, 16, 1887.

(18) Stein, R. S.; Onogi, S.; Keedy, D. J . Polym. Sci. 1962, 57, 801.

(19) Inoue, T.; Okamoto, H.; Osaki, K. Macromol ecules 1991, 24, 5670.

(20) Okamoto, H.; Inoue, T.; Osaki, K. J . Polym. Sci., Polym. Phys. Ed. 1995, 33, 417.

(21) Okamoto, H.; Inoue, T.; Osaki, K. J . Polym. Sci., Polym. Phys. Ed. 1995, 33, 1409.

(22) Osaki, K.; Okamoto, H.; I noue, T.; Hwang, E. Macromolecules 1995, 28, 3625.

(23) Flory, P.J . Statistical Mechanics of Chain Molecules; Oxford University Press: New York, 1969.

(24) J aneschitz-Kriegl, H. Polymer Mett Rheology and Flow Birefringence; Springer: New York, 1983.

(25) Roland, C. M.; Warzel, M. L. Rubber Chem. Technol. 1990, $63,285$.

(26) Baxandall, L. G.; Edwards, S. F. Macromolecules 1988, 21, 1763.

(27) Twardowski, T. E.; Gaylord, R. G. Polym. Bull. 1989, 21, 393.

(28) Santangelo, P. G.; Roland, C. M. Rubber Chem. Technol. 1995, 68, 124.

(29) Santangelo, P. G.; Roland, C. M. Rubber Chem. Technol. 1994, $67,359$.

(30) Andrews, R. O.; Kazama, Y. J . Appl. Phys. 1968, 39, 4891.

(31) Roland, C. M.; Kallitsis, J . K.; Gravalos, K. G. Macromolecules 1993, 26, 6474.

(32) Mott, P. H.; Roland, C. M. Rubber Chem. Technol. 1995, 68, 739.

(33) Gent, A. N.; Lindley, P. B. Proc. Inst. Mech. Eng. (London) 1959, 173, 111.
(34) Gent, A. N.; Henry, R. L.; Roxbury, M. L. J . Appl. Mech. 1974, $41,855$.

(35) Plazek, D. J .J . Polym. Sci., Polym. Chem. Ed. 1966, 4, 745.

(36) Roland, C. M. Rubber Chem. Technol. 1989, 62, 880.

(37) Rivlin, R. S.; Saunders, D. W. Phil. Trans. R. Soc. London, Ser. A 1951, 243, 251.

(38) Pak, H.; Flory, P. J . J . Polym. Sci., Polym. Phys. Ed. 1979, 17, 1845.

(39) Erman, B.; Flory, P. J . J . Polym. Sci., Polym. Phys. Ed. 1978, 16,1115

(40) J aneschitz-Kregl, H. Adv. Polym. Sci. 1969, 6, 170

(41) Ferry, J. D. Viscoelastic Properties of Polymers; Wiley: New York, 1980, p 573.

(42) Deng, D.; Argon, A. S.; Yip, S. Phil. Trans. R. Soc. London, Ser. A 1989, 329, 549, 575, 595, 613.

(43) Grosberg, A. Macromolecules 1993, 26, 3200.

(44) Duschl, E. J .; Goritz, D. Rubber Chem. Technol. 1991, 64, 668.

(45) Rooney, N. M.; von Hellens, C. W.; Tinker, A. J .; Brown, P. S. Rubber Chem. Technol. 1994, 67, 198

(46) Mendes, E.; Linder, P.; Buzier, M.; Boué, F.; Bastide, J . Phys. Rev. Lett. 1991, 66, 1595.

(47) Rouf, C.; Bastide, J .; Pujol, J . M.; Schosseler, F.; Munch, J . P. Phys. Rev. Lett. 1994, 73, 830.

(48) Yannas, I. V.J . Polym. Sci., Part D: Macromol. Rev. 1974, 9 163.

(49) Mullins, L. Rubber Chem. Technol. 1969, 42, 339.

(50) Harwood, J . A. C.; Payne, A. R. J . Appl. Polym. Sci. 1966, $10,315,1203$.

(51) Harwood, J . A. C.; Schallamach, A. J . Appl. Polym. Sci. 1967, $11,1835$.

(52) Roland, C. M. J . Rheol. 1989, 33, 659.

(53) Santangel o, P. G.; Roland, C. M. Rubber Chem. Technol. 1992, 65, 965.

(54) Larson, R. G. Constitutive Equations for Polymer Melts and Solutions; Butterworth: Boston, 1988.

(55) Wagner, M. H. J . Non-Newtonian Fluid Mech. 1978, 4, 39.

(56) Larson, R. G.; Valesano, V. A. J . Rheol. 1986, 30, 1093.

MA9608479 